\title{
Depressor Effect by Exercise Training Is Associated with Amelioration of Hyperinsulinemia and Sympathetic Overactivity
}

\author{
Keisuke Kohno, Hidehiro Matsuoka, Kenji TakenaKa, Yo Miyake, \\ Seiya OKuda, Gakuji Nomura and Tsutomu Imaizumi
}

\begin{abstract}
Objective In hypertensive subjects, exercise training is a therapeutic modality that not only lowers blood pressure but also corrects metabolic abnormality, such as hyperinsulinemia. Insulin causes sympatho-excitation via the modification of baroreflex, norepinephrine release, or central sympathetic outflow. However, the link between neural and metabolic changes by exercise training in hypertensive patients remains unknown. The aim of this study was to examine whether or not the blood pressure lowering effect of exercise training is associated with the improvement of insulin sensitivity in conjunction with the inhibition of sympathetic tone in hypertensive patients.

Methods We evaluated plasma insulin levels, arterial baroreflex function and humoral parameters before and after exercise training. Twenty-nine patients with essential hypertension under hospitalization participated in the study. Before and after three weeks of exercise training (75\% $\max \mathrm{VO}_{2}, 6$ min, q.i.d.), 24-hour blood pressure recordings, arterial baroreflex function testing and $75 \mathrm{~g}$ glucose tolerance tests were conducted. Area under the curve of insulin ( $\Sigma$ insulin) to glucose load was calculated as an index of hyperinsulinemia.

Results Three weeks of exercise training decreased the 24-hour mean arterial pressure, heart rate and $\Sigma$ insulin, and improved baroreflex function. There was a significant correlation between the reduction of arterial pressure and the change in $\Sigma$ insulin. Furthermore, the reduction of $\Sigma$ insulin was correlated with the improvement of baroreflex function and with the decrease in heart rate.

Conclusions Exercise training lowered the arterial pressure, with parallel changes in heart rate, baroreflex function and insulin resistance. The correction of sympathetic overactivity was closely associated with the amelioration of hyperinsulinemia. Our results suggest that the improvement of neuro-metabolic factors may be involved in the de-
\end{abstract}

pressor effect caused by exercise training. (Internal Medicine 39: 1013-1019, 2000)

Key words: baroreflex, essential hypertension, insulin, physical training, sympathetic nervous system

\section{Introduction}

The Joint National Committee (1) and World Health Organization/International Society of Hypertension (2) recommend exercise training as a primary therapy of hypertension. Although mechanisms underlying the blood pressure lowering effect of exercise training have not been clarified entirely (3-5), exercise training is widely recognized to correct metabolic abnormalities, such as hyperinsulinemia/insulin resistance $(6,7)$ which may contribute to its depressor effects.

Many studies have shown that essential hypertension is associated with insulin resistance (8-15). Although it remains unclear whether hyperinsulinemia/insulin resistance is a cause, a consequence or just an epiphenomenon of hypertension (13), we and others have recently demonstrated that non-obese nondiabetic subjects with hyperinsulinemia are at risk to develop hypertension in the future (16-19), suggesting the contributory role of hyperinsulinemia/insulin resistance in the pathogenesis of human hypertension. The most commonly proposed mechanisms for the link between hypertension and hyperinsulinemia/insulin resistance are: 1) insulin-induced antinatriuresis $(13), 2)$ altered vascular smooth muscle structure and function $(15), 3)$ interaction with endothelium-dependent relaxation and contraction (10), and 4) activation of the sympathetic nervous system $(9,14,20)$. Among the various factors mentioned above, activation of the sympathetic nervous system is indicated as a possible candidate for a number of reasons. Insulin causes sympathoexcitation (21) via the modification of baroreflex (22-24), of norepinephrine releases (25)

From the Department of Internal Medicine III and the Cardiovascular Research Institute, Kurume University School of Medicine, Fukuoka Received for publication December 6,1999; Accepted for publication May 30, 2000

Reprint requests should be addressed to Dr. Hidehiro Matsuoka, the Department of Internal Medicine III, Kurume University School of Medicine, 67 Asahimachi, Kurume, Fukuoka 830-0011 
and of the central sympathetic outflow (23). Furthermore, although insulin per se has a vasodilatory action $(10,26)$, sympathetic overactivity may antagonize the vasodilator actions of insulin (9).

Several lines of evidence have suggested that lowering sympathetic nerve activity may play a role in an exercise-induced reduction of blood pressure $(3-5,27)$. Indeed, exercise training not only lowers blood pressure but also decreases heart rate $(7,28,29)$ associated with increases in variabilities of heart rate $(28,29)$, decreases in the plasma norepinephrine concentration (30) and the norepinephrine spillover ratio (7), and improves arterial $(29,31)$ and cardiopulmonary $(32)$ baroreflex functions. However, since no study has evaluated changes in insulin sensitivity and sympathetic tone after exercise training in the same hypertensive subject, the link between neural and metabolic changes as a result of exercise training in hypertensives remains unknown.

Accordingly, to study whether the blood pressure lowering effect of exercise training is associated with the improvement of insulin sensitivity in conjunction with the inhibition of sympathetic tone in hypertensive patients, we evaluated plasma insulin levels, arterial baroreflex function and neuro-humoral parameters before and after exercise training.

\section{Patients and Methods}

\section{Study protocol}

A total of 29 patients with mild to moderate essential hypertension ( 17 men and 12 women) aged $43 \pm 2$ years were enrolled in this study. Informed consent was obtained and the study protocol based on our institutional ethics committee was approved. Subjects' blood pressure was measured weekly for 1 month before initiation of the study protocol. Hypertensive patients were qualified for the study if their systolic and/or diastolic blood pressure was $\geq 140$ and/or $90 \mathrm{mmHg}$, respectively on the last two visits of this period. Before enrolling them in the study, all the subjects were asked to report their daily activity. Only patients who were office workers or housewives having no regular exercise were enrolled. Secondary hypertension was ruled out by clinical and laboratory evaluations. Medications were gradually withdrawn and completely discontinued 2 weeks before the start of the study. Subjects were hospitalized during the study to control their physical activity and diet. To determine daily calorie intake, we adopted $65 \mathrm{~kg}$, average weight of a middle-aged Japanese, as standard body weight. Since total calorie intake of $25 \mathrm{kcal} / \mathrm{kg} / \mathrm{day}$ for hospitalized patients is recommended, we put them on a total calorie intake of approximately $1,600 \mathrm{kcal} / \mathrm{day}$. The patients were also put on a salt intake of less than $7 \mathrm{gram} /$ day, which has been commonly recommended for hypertensive patients in clinical practice. After the 2-week observation period in the hospital, we measured 24-hour blood pressure, heart rate, and exercise capacity within a week before and after a 3-week exercise training program. In some patients who agreed to further examinations, we evaluated insulin sensitivity, arterial baroreflex function and humoral parameters.

\section{Training program}

Subjects performed 4 sessions of cycle ergometer exercise daily for 3 weeks. Each exercise session consisted of a 3-minute warm-up period, a 6-minute period of exercise at an intensity corresponding to $75 \%$ of the maximal oxygen uptake determined by analysis of expired gases, and a 3-minute cool-down period as previously described (33). The aerobic exercise intensity was determined by a multistage graded exercise test on a treadmill before and after exercise training.

\section{Twenty-four-hour blood pressure and heart rate}

An automatic non-invasive recorder (ABPM-630, NihonColin) (34) was used to measure 24-hour blood pressure and heart rate. Measurements were made at 15 -minute intervals between 6:00 AM and 9:00 PM (daytime) and at 30-minute intervals between 9:00 PM and 6:00 AM (nighttime). Patients were asked to relax their arms during cuff inflation and deflation. After the 24-hour recordings were obtained, the data were downloaded from the recorder to a computer for analysis. We excluded the following values from analysis: systolic pressures below 70 or above $250 \mathrm{mmHg}$, diastolic pressures below 40 or above $150 \mathrm{mmHg}$, and heart rates below 40 or above 150 beats per minute. We calculated standard deviation of 24-hour, and day- and night-time blood pressure and heart rate as indices of pressure and heart rate variabilities, respectively.

\section{Insulin responses to glucose load}

Insulin sensitivity was determined by an oral glucose tolerance test with 75 gram glucose. The $75-\mathrm{g}$ oral glucose tolerance test (75-g OGTT) was performed in the morning after overnight fasting. Blood samples were taken at $0,30,60$, and 120 minutes after glucose administration to measure plasma glucose and insulin. Area under the curves of plasma insulin ( $\Sigma$ insulin) and plasma glucose ( $\Sigma$ glucose) during the 2-hour 75-g OGTT were calculated using the trapezoidal rule and the ratio of $\Sigma$ insulin to $\Sigma$ glucose during 75-g OGTT was used as an index of insulin resistance (35). Diabetes mellitus, diagnosed according to the criteria of the American Diabetes Association (36), defined as a fasting glucose level of $126 \mathrm{mg} / \mathrm{dl}$ or greater and/or plasma glucose level of $200 \mathrm{mg} / \mathrm{dl}$ or greater at 2 hours after glucose administration, was excluded from this study. Based on this criteria, there were 8 patients with impaired glucose tolerance.

\section{Assessment of baroreflex function}

Beat-to-beat R-R intervals and corresponding systolic and diastolic blood pressure were collected simultaneously over a 12- to 15-min period using a cardiotachometer and Finapres monitor, respectively. Arterial baroreflex function was assessed by determining the relationship between the changes in systolic blood pressure and the changes in the R-R interval induced by amyl nitrate inhalation by regression analysis, using a modification of the method by Smyth et al (37). Only slopes with regression coefficients $>0.9$ were included in the data analysis. 


\section{Measurement of humoral factors}

After the overnight fasting, blood samples were withdrawn from the antecubital vein into vacuum tubes after 30-minute bed rest, immediately placed on ice, and centrifuged at $1,800 \mathrm{~g}$ at $4^{\circ} \mathrm{C}$. Plasma was stored at $-80^{\circ} \mathrm{C}$ until used for analyses. The plasma concentration of norepinephrine (PNE) was determined by high-performance liquid chromatography with electrochemical detection (HLC-725CA, Tohso). Radioimmunoassay kits were used to determine the plasma insulin level (Pharmacia Insulin RIA kit, Pharmacia KK, Japan), and the plasma renin activity (PRA; Gamma Coat, Baxter, U.S.A.). Plasma glucose was measured by the glucose dehydrogenase ultraviolet test (Merck Liquid Glu, Kanto Chemical Co., Japan).

\section{Statistical analysis}

Results are expressed as the mean \pm SEM. The effects of ex- ercise training on variables were examined by two-tailed paired Student's $t$-test. A probability of $<0.05$ was accepted as the level of statistical significance. Correlation analyses were performed by linear regression analysis.

\section{Results}

\section{Effects of exercise training on body weight, oxygen uptake (Table 1), blood pressure and heart rate (Table 2)}

The maximal oxygen uptake increased $(\mathrm{p}<0.05)$, and body weight decreased significantly $(\mathrm{p}<0.0001)$ after the 3 -week training program, although there was no correlation between the degree of body weight reduction and the changes in hemodynamic or neuro-humoral parameters described as follows. Exercise training significantly reduced systolic blood pressure $(\mathrm{p}<0.001$ ), and mean (from $104 \pm 2$ to $101 \pm 2 \mathrm{mmHg}, \mathrm{p}<0.05$ ) arterial pressure and tended to decrease diastolic $(\mathrm{p}=0.08)$ blood

Table 1. Body Weight, BMI, $\mathrm{VO}_{2}, \mathrm{BSI}, \mathrm{P}-\mathrm{NE}$ and PRA before and after Exercise Training

\begin{tabular}{lccc}
\hline & before & after & before vs after \\
\hline Body weight $(\mathrm{kg})$ & $66.0 \pm 1.5$ & $64.1 \pm 1.4$ & $\mathrm{p}<0.0001$ \\
$\mathrm{BMI}\left(\mathrm{kg} / \mathrm{m}^{2}\right)$ & $25.6 \pm 0.7$ & $24.8 \pm 0.6$ & $\mathrm{p}<0.0001$ \\
$\mathrm{VO}_{2}(\mathrm{ml} / \mathrm{kg} / \mathrm{min})$ & $25.1 \pm 1.4$ & $27.3 \pm 1.9$ & $\mathrm{p}<0.05$ \\
$\mathrm{BSI}(\mathrm{msec} / \mathrm{mmHg})$ & $6.34 \pm 0.6$ & $8.05 \pm 1.0$ & $\mathrm{p}<0.05$ \\
Plasma norepinephrine $(\mathrm{ng} / \mathrm{dl})$ & $0.23 \pm 0.02$ & $0.21 \pm 0.02$ & $\mathrm{NS}$ \\
Plasma renin activity $(\mathrm{ng} / \mathrm{ml} / \mathrm{h})$ & $1.36 \pm 0.18$ & $1.09 \pm 0.14$ & $\mathrm{p}<0.05$ \\
\hline
\end{tabular}

BMI: body mass index, $\mathrm{VO}_{2}$ : maximal oxygen uptake, BSI: arterial baroreceptor function.

Table 2. Hemodynamics before and after Exercise Training

\begin{tabular}{|c|c|c|c|c|}
\hline & & before & after & before vs after \\
\hline \multicolumn{5}{|c|}{ Systolic blood pressure (mmHg) } \\
\hline \multirow[t]{2}{*}{ All day } & mean & $135.9 \pm 2.7$ & $131.0 \pm 2.7$ & $\mathrm{p}<0.001$ \\
\hline & SD & $17.6 \pm 1.0$ & $15.9 \pm 0.7$ & NS \\
\hline \multirow[t]{2}{*}{ Daytime } & mean & $139.8 \pm 2.8$ & $134.1 \pm 2.8$ & $\mathrm{p}<0.0005$ \\
\hline & $\mathrm{SD}$ & $16.5 \pm 1.0$ & $14.9 \pm 0.7$ & NS \\
\hline \multirow[t]{2}{*}{ Nighttime } & mean & $122.6 \pm 2.7$ & $119.9 \pm 2.8$ & NS \\
\hline & $\mathrm{SD}$ & $13.5 \pm 1.2$ & $12.1 \pm 0.8$ & NS \\
\hline \multicolumn{5}{|c|}{ Diastolic blood pressure (mmHg) } \\
\hline \multirow[t]{2}{*}{ All day } & mean & $88.6 \pm 1.9$ & $86.7 \pm 1.8$ & NS \\
\hline & $\mathrm{SD}$ & $12.0 \pm 0.5$ & $12.3 \pm 1.0$ & NS \\
\hline \multirow[t]{2}{*}{ Daytime } & mean & $91.0 \pm 1.9$ & $88.9 \pm 2.8$ & NS \\
\hline & $\mathrm{SD}$ & $11.5 \pm 0.5$ & $12.1 \pm 1.2$ & NS \\
\hline \multirow[t]{2}{*}{ Nighttime } & mean & $80.0 \pm 1.8$ & $79.1 \pm 1.7$ & NS \\
\hline & SD & $8.0 \pm 0.5$ & $13.5 \pm 0.5$ & NS \\
\hline \multicolumn{5}{|c|}{ Heart rate $(\mathrm{bpm})$} \\
\hline \multirow[t]{2}{*}{ All day } & mean & $70.7 \pm 1.6$ & $68.2 \pm 1.2$ & $\mathrm{p}<0.05$ \\
\hline & $\mathrm{SD}$ & $12.0 \pm 0.5$ & $11.4 \pm 0.6$ & NS \\
\hline \multirow[t]{2}{*}{ Daytime } & mean & $73.6 \pm 1.7$ & $71.4 \pm 1.4$ & NS \\
\hline & $\mathrm{SD}$ & $11.3 \pm 0.5$ & $10.3 \pm 0.6$ & NS \\
\hline \multirow[t]{2}{*}{ Nighttime } & mean & $60.9 \pm 1.8$ & $57.8 \pm 1.2$ & $\mathrm{p}<0.05$ \\
\hline & SD & $6.3 \pm 0.6$ & $7.1 \pm 0.9$ & NS \\
\hline
\end{tabular}

Daytime, 6:00AM-9:00PM; Nighttime, 9:00PM-6:00AM. 


\section{KoHNo et al}

pressure measured during 24-hours. The effect of training was more pronounced in daytime systolic blood pressure $(p<0.0005)$. Twenty-four hour averaged heart rate also decreased after exercise training $(\mathrm{p}<0.05)$.

\section{Effects of exercise training on blood pressure variability (Table 2)}

Standard deviations of blood pressure and heart rate obtained by 24-hour recordings were calculated as indices of variabilities of blood pressure and heart rate, respectively. Although systolic blood pressure variability in 24 hours tended to be reduced after exercise training ( $\mathrm{p}=0.07)$, the diastolic pressure and heart rate variabilities did not change significantly after exercise training.

\section{Change in arterial baroreflex function (Table 1)}

Before exercise, arterial baroreflex function was inversely correlated with 24-hour systolic pressure $(r=-0.44, \mathrm{p}<0.05)$ and with systolic pressure variability $(r=-0.56, p<0.05)$. Exercise training significantly improved arterial baroreflex function $(\mathrm{p}<0.05)$. The improvement of arterial baroreflex function was significantly correlated with 24 -hour heart rate reduction $(\mathrm{r}=0.56, \mathrm{p}<0.05)$ and this correlation was more pronounced in the daytime heart rate $(\mathrm{r}=0.63, \mathrm{p}<0.005)$.

\section{Change in plasma norepinephrine (Table 1)}

PNE tended to be decreased by exercise training but not significantly. The decrease in PNE was significantly correlated with the reduction in 24-hour systolic blood pressure $(r=0.50$, $\mathrm{p}<0.05$; Fig. $1 \mathrm{~A}$ ) and tended to be correlated with the decrease in 24-hour heart rate $(r=0.37, p=0.08$; Fig. 1B). There was an inverse relationship between the PNE reduction and the improvement of arterial baroreflex function significantly $(\mathrm{r}=-0.52$, p $<0.05$; Fig. 1C).

\section{Change in plasma renin activity (Table 1)}

PRA significantly decreased after exercise training $(\mathrm{p}<0.05)$. The decrease in PRA was correlated with the improvement of arterial baroreflex function $(r=0.47, p<0.05)$.

\section{Change in insulin responses to glucose (Table 3)}

Prior to exercise training, the fasting plasma insulin level was positively correlated with PNE $(r=0.57, \mathrm{p}<0.05)$ and inversely correlated with arterial baroreflex function $(r=-0.65$, $\mathrm{p}<0.05$ ). Significant correlations were observed between PNE and the 120-min insulin level $(\mathrm{r}=0.47, \mathrm{p}<0.05)$ or $\Sigma$ insulin $(\mathrm{r}=0.46, \mathrm{p}<0.05)$. Exercise training significantly reduced plasma insulin levels at 60 minutes $(p<0.005)$ and 120 minutes $(p<0.05)$ after oral glucose loading, resulting in a marked reduction of $\Sigma$ insulin $(\mathrm{p}<0.005)$. The plasma glucose levels at fasting $(\mathrm{p}<0.05)$, 30 minutes $(p<0.01)$, and 60 minutes $(p<0.01)$ were significantly decreased, resulting in a marked reduction of $\Sigma$ glucose $(\mathrm{p}<0.005)$ after the training. The ratio of $\Sigma$ insulin to $\Sigma$ glucose also significantly decreased after the training $(\mathrm{p}<0.05)$. The reduction of $\Sigma$ insulin was correlated with the decrease in the 24-hour mean arterial pressure $(r=0.53, p<0.05 ;$ Fig. $2 \mathrm{~A})$ and with the decrease in the 24-hour heart rate $(r=0.57, p<0.05$; Fig. 2B). The reduction of $\Sigma$ insulin was also correlated with the improvement of arterial baroreflex function $(r=-0.64$, $\mathrm{p}<0.05$; Fig. 2C), and with the decrease in PRA $(r=0.52$, $\mathrm{p}<0.05)$.

\section{Discussion}

The major findings of this study were: a) 3 weeks of exercise training reduced the 24-hour mean arterial pressure and heart rate, and improved arterial baroreflex function and hyperinsulinemia, b) there was a significant correlation between the blood pressure reduction and the improvement of hyperinsulinemia. c) the improvement of hyperinsulinemia was

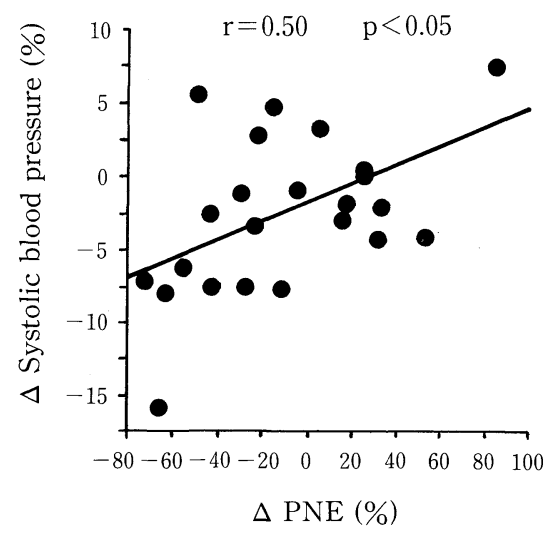

A

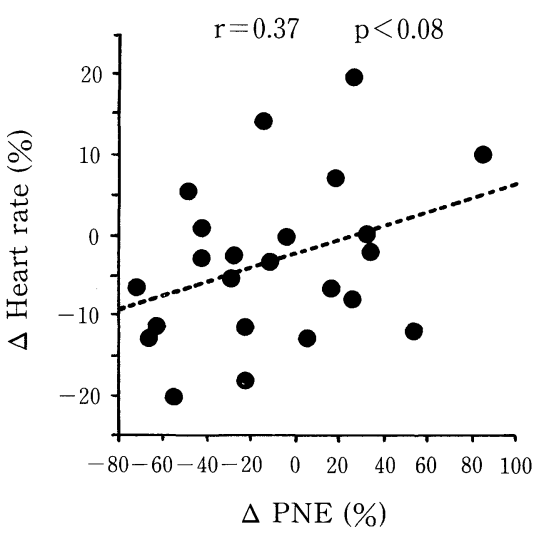

$\mathrm{B}$

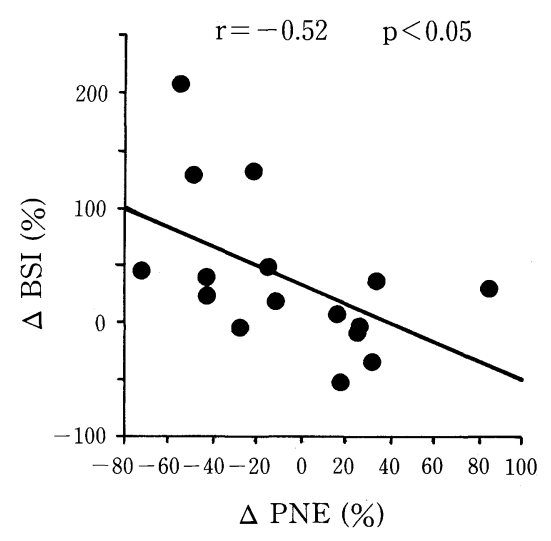

$\mathrm{C}$

Figure 1. The relationships between the plasma norepinephrine concentration (PNE) and 24-hour systolic blood pressure (n=23) (A), 24-hour heart rate $(n=23)(B)$, or arterial baroreflex function (BSI) $(n=16)(C)$, with 3weeks of exercise training. All data are expressed as percent changes from baseline values before exercise. 
Table 3. 75-g Oral Glucose Tolerance Test before and after Exercise Training

\begin{tabular}{lrccc}
\hline & & before & after & before vs after \\
\hline Glucose $(\mathrm{mg} / \mathrm{dl})$ & & & & \\
$\quad$ Time after glucose intake & $0 \mathrm{~min}$ & $90.9 \pm 2.0$ & $88.2 \pm 1.9$ & $\mathrm{p}<0.05$ \\
& $30 \mathrm{~min}$ & $157.9 \pm 6.4$ & $139.8 \pm 5.8$ & $\mathrm{p}<0.01$ \\
& $60 \mathrm{~min}$ & $157.4 \pm 10.8$ & $133.9 \pm 9.5$ & $\mathrm{p}<0.01$ \\
& $120 \mathrm{~min}$ & $126.2 \pm 5.5$ & $114.6 \pm 4.9$ & $\mathrm{NS}$ \\
$\Sigma$ glucose $(\mathrm{mg} / \mathrm{dl} \cdot \mathrm{h})$ & & $282.3 \pm 13.3$ & $249.7 \pm 11.2$ & $\mathrm{p}<0.005$ \\
Immunoreactive insulin & & & & \\
$(\mu \mathrm{U} / \mathrm{ml})$ & & & & \\
$\quad$ Time after glucose intake & $0 \mathrm{~min}$ & $9.1 \pm 1.3$ & $8.9 \pm 1.3$ & $\mathrm{NS}$ \\
& $30 \mathrm{~min}$ & $82.8 \pm 9.0$ & $64.7 \pm 10.2$ & $\mathrm{NS}$ \\
& $60 \mathrm{~min}$ & $86.7 \pm 11.2$ & $59.4 \pm 9.5$ & $\mathrm{p}<0.005$ \\
& $120 \mathrm{~min}$ & $87.0 \pm 16.2$ & $61.3 \pm 14.8$ & $\mathrm{p}<0.05$ \\
$\Sigma$ insulin $(\mu \mathrm{U} / \mathrm{ml} \cdot \mathrm{h})$ & & $149.0 \pm 18.1$ & $109.7 \pm 16.8$ & $\mathrm{p}<0.005$ \\
$\Sigma$ insulin $/ \Sigma$ glucose & & $0.53 \pm 0.06$ & $0.42 \pm 0.05$ & $\mathrm{p}<0.05$ \\
\hline
\end{tabular}

$\Sigma$ glucose and $\Sigma$ insulin indicate areas composed of glucose and insulin levels, respectively at $0,30,60$, 120 minutes after glucose load.
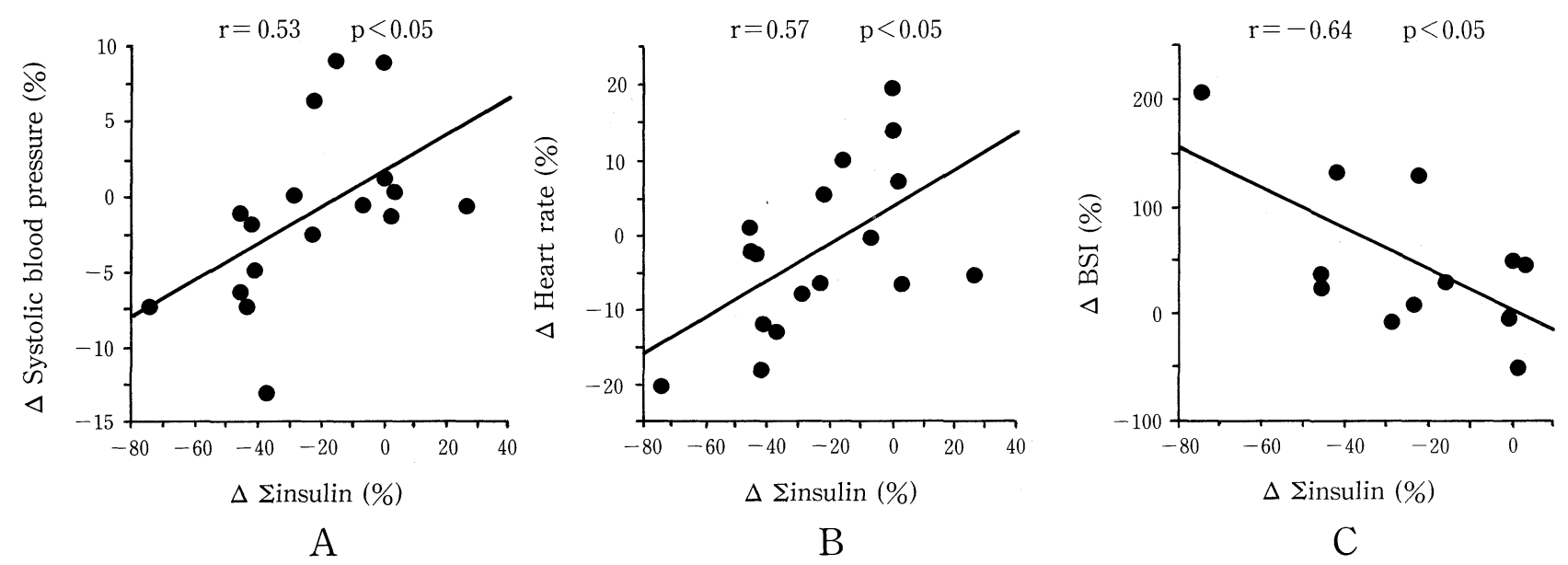

Figure 2. The relationships between $\Sigma$ insulin and 24-hour mean arterial pressure $(n=17)(A), 24-h o u r$ heart rate $(n=17)(B)$, or arterial baroreflex function $(B S I)(n=13)(C)$, with 3 weeks of exercise training. All data are expressed as percent changes from baseline values before exercise.

correlated with the improvement of arterial baroreflex function and with the decrease in heart rate. Thus we demonstrated that the blood pressure lowering effect of exercise training is associated with improvement of insulin sensitivity in conjunction with the inhibition of sympathetic tone in hypertensive patients.

In the present study, we adopted a special exercise protocol according to our previous report (33). Exercise training was performed on an inpatient basis so that the patients' physical activity and diet could be strictly controlled. Exercise capacity increased, body weight and blood pressure decreased, and glucose tolerance improved after 3-weeks of short multiple bouts of exercise. The significant increase in $\mathrm{VO}_{2}$ max by exercise training indicates that our training protocol was effectively performed. It is unlikely that changes in hemodynamic or neurohumoral parameters by exercise training were attributed to body weight reduction per se, since there was no correlation between the degree of body weight reduction and the changes in hemodynamic or neurohumoral parameters in our study.

Area under the curve of insulin ( $\Sigma$ insulin) and that of glucose ( $\Sigma$ glucose) were calculated and the ratio of $\Sigma$ insulin to $\Sigma$ glucose during 75-g OGTT was used as an index of insulin resistance (35) in our study, because the insulin level is determined by the glucose level and insulin resistance (38). Several other factors such as the pancreatic secretory capacity of insulin, previous activity level, gastrointestinal motility, and nutritional status also determine the insulin level (38). Because we measured the insulin level before and after exercise training in 


\section{KoHNO et al}

the same individual, it was unlikely that the pancreatic secretory capacity of insulin, previous activity level, and gastrointestinal motility were altered after exercise training. Thus the decreased insulin level after exercise training in this study could have been due to improved insulin sensitivity and/or the decrease in the glucose level. The reduction of $\Sigma$ insulin was significantly greater than that of $\Sigma$ glucose, resulting in the decrease in $\Sigma$ insulin $/ \Sigma$ glucose after exercise training. Thus, consistent with previous studies $(6,7)$, the present results indicated that exercise training improved not only hyperinsulinemia but also insulin sensitivity.

The mechanisms potentially responsible for blood pressure reduction by exercise training have not been elucidated entirely $(3-5,27)$. Candidates range from afferent, central or peripheral nervous modifications (39-41) and hormonal factors such as the renin-angiotensin system $(7,42,43)$ to structural and functional modifications of the heart and vasculature mediated by systemic or local factors (44) have been suggested to contribute to the blood pressure lowering effect of exercise training. Among them, it has been demonstrated that the exerciseinduced blood pressure lowering effect is, at least partially mediated by the correction of sympathetic overactivity $(9,14,20)$. Decreases in plasma norepinephrine $(7,42,43)$ and the peripheral sympathetic nerve activity (45) after exercise have been reported. Consistent with previous reports, here 3 weeks of exercise training reduced 24 -hour blood pressure as well as 24-hour heart rate. The blood pressure lowering effect was more pronounced during the daytime, during which subjects have higher sympathetic nerve activity. Furthermore, exercise training ameliorated arterial baroreflex function. The reduction of blood pressure was significantly correlated with the reduction of plasma norepinephrine. Collectively, the decrease in sympathetic nerve activity was associated with the blood pressure reduction by exercise training.

Since exercise training improves metabolic abnormalities such as hyperinsulinemia/insulin resistance, in which sympathetic overactivity has been demonstrated, we thought that correction of hyperinsulinemia/insulin resistance might contribute to the sympathoinhibition, and hence to the blood pressure lowering effect of exercise training. Indeed, exercise training improved insulin sensitivity in parallel with the reduction of blood pressure, and with the improvement of arterial baroreflex function. Therefore, it is possible that exercise-induced improvement of insulin sensitivity was associated with the decrease in sympathetic nerve activity and with the blood pressure reduction in hypertensive subjects.

In summary, 3 weeks of exercise training lowered the arterial pressure, with parallel changes in the heart rate, baroreflex function and insulin resistance. The correction of sympathetic overactivity was closely associated with the amelioration of hyperinsulinemia. The present results suggest that the improvement of neuro-metabolic factors may be involved in the depressor effect which results from exercise training.

Acknowlegements: This study was supported in part by a Grant-in-Aid for Scientific Research from the Ministry of Education, Science and Culture
(\#2-635-6039-07770547 and \#2-635-7036-08770533) and by a grant from Kimura Memorial Heart Foundation, Kurume, Japan. The authors are indebted to Tamami Iguchi and Shino Yamakawa for their excellent technical assistance.

\section{References}

1) The sixth report of the Joint National Committee on prevention, detection, evaluation, and treatment of high blood pressure (JNC VI). Arch Intern Med 158: 2413-2446, 1997.

2) 1999 World Health Organization-International Society of Hypertension Guidelines for the Management of Hypertension. Guidelines Subcommittee. J Hypertens 17: 151-183, 1999.

3) Hagberg JM. Exercise, fitness and hypertension, in: Bouchard C SR, Stephens T, Sutton JR, McPherson BD, Eds. Exercise, Fitness, and Health A Consensus of Current Knowledge. Human Kinetics Books, Champaign, 1990: 455-466.

4) Arakawa K. Antihypertensive mechanism of exercise. J Hypertens 11: 223-229, 1993.

5) Jennings GL, Kingwell BA. Exercise. In: Swales JD, Ed. Textbook of Hypertension. Blackwell Scientific Publications, London, 1994: 593-604.

6) Rosenthal M, Haskell WL, Solomon R, Widstrom A, Reaven GM. Demonstration of a relationship between level of physical training and insulin-stimulated glucose utilization in normal humans. Diabetes 32: 408$411,1983$.

7) Jennings G, Nelson L, Nestel P, et al. The effects of changes in physical activity on major cardiovascular risk factors, hemodynamics, sympathetic function, and glucose utilization in man: a controlled study of four levels of activity. Circulation 73: 30-40, 1986.

8) Ferrannini E, Haffner SM, Stern MP. Essential hypertension: An insulin resistant state. J Cardiovasc Pharmacol 15 (Suppl 5): S18-S25, 1990.

9) Julius S, Gudbrandsson T, Jamerson K, Shahab ST, Anderson O. The hemodynamic link between insulin resistance and hypertension. J Hypertens 9: 983-986, 1991.

10) Baron AD. Hemodynamic actions of insulin. Am J Physiol 267: E187E202, 1994.

11) Morris AD, Petrie J R, Connell JM. Insulin and hypertenion. J Hypertens 12: 633-642, 1994.

12) Shimamoto $K$, Hirata $A$, Fukuoka M, Iimura O. Insulin sensitivity and the effects of insulin on renal sodium handling and pressor systems in essential Hypertensive patients. Hypertension 23 (Suppl I): I-29-I-33, 1994.

13) Hall JE, Brands MW, Zappe DH, Galicia MA. Insulin resistance, hyperinsulinemia, and hypertension: causes, consequences, or merely correlations ? Proc Soc Exp Biol Med 208: 317-329, 1995.

14) Moreau P, Lamarche L, Laflamme AK, Calderone A, Yamaguchi N, de Champplain J. Chronic hyperinsulinemia and hypertension: The role of the sympathetic nervous system. J Hypertens 13: 333-340, 1995.

15) Reaven GM, Lithell H, Landsberg L. Hypertension and assiciated metabolic abnormalities: the role of insulin resistance and the sympathoadrenal system. N Engl J Med 334: 374-381, 1996.

16) Skarfors ET, Lithell HO, Selinus I. Risk factors for the development of hypertension: A 10-year longitudinal study in middle-aged men. $\mathbf{J}$ Hypertens 9: 217-223, 1991.

17) Haffner SM, Valdez RA, Hazuda HP, Mitchell BD, Morales PA, Stern MP. Prospective analysis of the insulin-resistance (Syndrome X). Diabetes 41: 715-722, 1992.

18) Lissner L, Bengstsson C, Lapidus L, Kristjansson K, Wedel H. Fasting insulin in relation to subsequent blood pressure changes and hypertension in women. Hypertension 20: 797-801, 1992.

19) Tsuruta $M$, Hashimoto $R$, Adachi H, Imaizumi T, Nomura G. Hyperinsulinemia as a predictor of hypertension: An 11-year follow-up study in Japan. J Hypertens 14: 483-488, 1996.

20) Muntzel MS, Anderson EA, Johnson AK, Mark AL. Mechanisms of insulin action on sympathetic nerve activity. Clin Exp Hypertens 17: 39$50,1995$. 


\section{Changes in Neuro-metabolic Factors by Exercise}

21) Anderson EA, Hoffman RP, Balon TW, Sinkey CA, Mark AL. Hyperinsulinemia produces both sympathetic neural activation and vasodilation in normal humans. J Clin Invset 87: 2246-2252, 1991.

22) Fitzovich DE, Randall DC. Modulation of baroreflex by varying insulin and glucose in conscious dogs. Am J Physiol 258: R624-R633, 1990.

23) Muntzel MS, Morgan DA, Mark AL, Johnson AK. Intracerebroventricular insulin produces nonuniform regional increases in sympathetic nerve activity. Am J Physiol 267: R1350-R1355, 1994.

24) Okada $M$, Bunag $R D$. Insulin acts centrally to enhance reflex tachycardia in conscious rats. Am J Physiol 266: R481-486, 1994.

25) Edwards JG, Tipton CM. Influences of exogenous insulin on arterial blood pressure measurements of the rat. J Appl Physiol 67: 2335-2342, 1989.

26) Sakai $K$, Imaizumi $T$, Masaki $H$, Takeshita $A$. Intra-arterial infusion of insulin attenuates vasoreactivity in human forearm. Hypertension 22: 6773, 1993.

27) Fagard R, Amery A. Physical exercise in hypertension. in: Laragh JH, Brenner BM, Eds Hypertension. Pathophysiology, Diagnosis, and Management. 2nd ed. Raven Press, New York, 1995: 2669-2682.

28) Pagani M, Somers V, Furlan R, et al. Changes in autonomic regulation induced by physical training in mild hypertension. Hypertension 12: 600$610,1988$.

29) Somers VK, Conway J, Johnson J, Sleight P. Effects of endurance training on baroreflex sensitivity and blood pressure in borderline hypertension. Lancet 337: 1363-1368, 1991.

30) Duncan JV, Farr JE, Upton ST, Hagan RD, Oglesby ME, Blair SN. The effects of aerobic exercise on plasma catecholamines and blood pressure in patients with mild essential hypertension. JAMA 254: 2609-2613, 1985.

31) Grassi G, Seravalle G, Calhoun D, Bolla GB, Mancia G. Physical exercise in essential hypertension. Chest 101: 312S-314S, 1992.

32) Takeshita A, Jingu S, Imaizumi T, Kunihiko Y, Koyanagi S, Nakamura $M$. Augmented cardiopulmonary baroreflex control of forearm vascular resistance in young athletes. Circ Res 59: 43-48, 1986.

33) Kohno K, Matsuoka H, Takenaka K, Miyake Y, Nomura G, Imaizumi T.
Renal depressor mechanisms of physical training in patients with essential hypertension. Am J Hypertens 10: 859-868, 1997.

34) White WB, Lund-Johansen P, McCabe EJ. Clinical evaluation of the Colin ABPM 630 at rest and during exercise: An ambulatory blood pressure monitor with gas-powered cuff inflation. J Hypertens 9: 477-483, 1987.

35) Bergman R, Finegood DT, Ader M. Assessment of insulin sensitivity in vivo. Endocr Rev 6: 45-86, 1985.

36) Report of the Expert Committee on the Diagnosis and Classification of Diabetes Mellitus. Diabetes Care 20:1183-1197, 1997.

37) Smyth HS, Sleight P, Pickering GW. Reflex regulation of arterial pressure during sleep in man. A quantitative method of assessing baroreflex sensitivity. Circ Res 24: 109-121, 1969.

38) Baron AD. Pathogenesis and measurement of insulin resistance in hypertension. Curr Opin Nephrol Hypertens 3: 631-635, 1994.

39) Björntorp P. Hypertension and exercise. Hypertension 4 (Suppl 3): 56$59,1982$.

40) Cleroux J, Peronnet F, de Champlain J. Sympathetic indices during psychological and physical stimuli before and after training. Physiol Behav 35: 271-275, 1985.

41) Grassi G, Seravalle G, Calhoun DA, Bolla GB, Mancia G. Physical training and baroreceptor control of sympathetic nerve activity in humans. Hypertension 23: 294-301, 1994.

42) Fagard R, Grauwels R, Groeseneken D, et al. Plasma levels of renin, angiotensin II and 6-keto-prostaglandin F1 $\alpha$ in endurance athletes. J Appl Physiol 59: 947-952, 1985.

43) Kiyonaga A, Arakawa K, Tanaka H, Shindo M. Blood pressure and humoral responses to aerobic exercise. Hypertension 7: 125-131, 1985.

44) Wang J, Wolin MS, Hintze TH. Chronic exercise enhances endotheliummediated dilatation of epicardial coronary artery in conscious dogs. Circ Res 73: 829-838, 1993.

45) Meredith IT, Friberg P, Jennings GJ, et al. Exercise training lowers resting renal but not cardiac sympathetic activity in humans. Hypertension 18: 575-582, 1991. 